\title{
Cosmological space-times with resolved Big Bang in Yang-Mills matrix models
}

\author{
Harold C. Steinacker \\ Faculty of Physics, University of Vienna, \\ Boltzmanngasse 5, A-1090 Vienna, Austria \\ E-mail: harold.steinacker@gmail.com
}

ABSTRACT: We present simple solutions of IKKT-type matrix models that can be viewed as quantized homogeneous and isotropic cosmological space-times, with finite density of microstates and a regular Big Bang (BB). The BB arises from a signature change of the effective metric on a fuzzy brane embedded in Lorentzian target space, in the presence of a quantized 4-volume form. The Hubble parameter is singular at the BB, and becomes small at late times. There is no singularity from the target space point of view, and the brane is Euclidean "before" the BB. Both recollapsing and expanding universe solutions are obtained, depending on the mass parameters.

Keywords: M(atrix) Theories, Non-Commutative Geometry, Spacetime Singularities

ArXiv EPrint: 1709.10480 


\section{Contents}

1 Introduction 1

2 Lorentzian matrix models 3

3 Recollapsing universe from fuzzy 4-spheres $\quad 4$

3.1 The Euclidean fuzzy 4-sphere 4

3.2 Lorentzian fuzzy 4-sphere in $\mathbb{R}^{1,4}$

4 Expanding universe from fuzzy hyperboloids $\quad 11$

$\begin{array}{lll}4.1 & \text { Euclidean fuzzy hyperboloids } & 11\end{array}$

$\begin{array}{lll}4.2 & \text { Lorentzian fuzzy hyperboloids } & 12\end{array}$

$\begin{array}{lll}4.3 \text { Outlook } & 17\end{array}$

$\begin{array}{llr}5 & \text { Discussion } & 18\end{array}$

A No finite-dimensional solutions of massless Lorentzian Yang-Mills matrix models 20

\section{Introduction}

The evolution of the universe and its origin in a Big Bang (BB) appear to be well described by the $\Lambda \mathrm{CDM}$ model of inflationary cosmology. This model is based on general relativity (GR), assuming suitable matter content and initial conditions. Nevertheless, the situation is not satisfactory. The model requires a dominant role of unknown matter and energy, while postulating that GR still applies at cosmological scales. At very short distances, GR quite certainly breaks down, and a quantum theory of gravity must take over. This is essential to address the local and global singularities of space-time, in particular the BB, but it also leads to serious fine-tuning problems.

There are strong reasons to expect that in a consistent quantum theory including gravity, there should be only finitely many degrees of freedom per unit "volume". While we do not know the correct micro-structure of space-time, it requires a pre-geometric origin of space-time. This also seems to be the most reasonable way to resolve the singularities in black holes and the BB.

Among the many possible approaches to this issue, we will follow an approach based on matrix models. By their very nature as discrete pre-geometric models, they provide natural candidates to address the above issues. Among all pure matrix models, the IKKT model [1] is singled out by virtue of maximal supersymmetry, and it was proposed as a candidate for a non-perturbative description of IIB string theory. Although there is at present no solid understanding of this model at a non-perturbative, background-independent level, there is 
a good picture of branes arising as classical solutions, with IIB supergravity interactions arising at the loop level [1-4]. The effective geometry of such branes (given by some matrix background ${ }^{1}$ ) can be elaborated as noncommutative or semi-classical geometry, and fluctuations of these backgrounds lead to noncommutative gauge theory coupled to this geometry $[5,6]$. Here the maximal supersymmetry of the matrix model plays an important role, since otherwise unacceptable large non-local effects due to UV/IR mixing $[4,7,8]$ ) invalidate the semi-classical picture.

In this paper, we will present explicit and simple brane solutions of the IKKT matrix model with mass term, which can serve as (toy-) models for cosmological space-times, and exhibit a BB-like singularity. They have a space-like $\mathrm{SO}(4)$ isometry, and reduce to homogeneous and isotropic FRW cosmologies with $k=1$ in the semi-classical limit. These solutions are obtained from basic quantized ("fuzzy") homogeneous spaces, specifically the fuzzy 4-sphere $S_{N}^{4}$ and the fuzzy 4-hyperboloid $H_{n}^{4}$. These turn out to be solutions of the Lorentzian matrix model in the presence of suitable mass terms, which are different for the space-like and time-like matrices. The BB arises from a signature change in the effective metric, taking into account the quantized 4-volume form which arises from the non-commutative structure of the brane. The point is that the effective metric on the brane $\mathcal{M}$ is not the induced metric, but involves the Poisson structure on the brane in an essential way ${ }^{2}$ [9-11]. The Poisson structure gives rise to the frame bundle, and its flux provides the measure for the integration on $\mathcal{M}$. This determines the conformal factor of the metric which is singular at the location of signature change, leading to a singular initial expansion.

It is well-known that fuzzy spaces can be solutions of Lorentzian matrix models, cf. [1217]. Even compact solutions were found in $[18,19]$, where it was pointed out that the induced metric on the brane can change from Euclidean to Minkowski signature. However, this alone is not sufficient to obtain a Big Bang, and it does not imply a rapid expansion. The present work differs from the previous ones in two important ways. First, we obtain 3+1-dimensional space-time solutions which are completely homogeneous and isotropic; more precisely, they are covariant under $\mathrm{SO}(4)$ acting on the spatial $S^{3}$. Second and most remarkably, a BB with rapid (singular) initial expansion is shown to arise automatically on these solutions. These space-times are governed not by GR but by the matrix model.

We provide two basic examples of such cosmological matrix space-times with BB,one describing a recollapsing universe with a big crunch,and one which is expanding forever. Although neither seems to agree very well with the standard cosmology (at least under the present crude analysis), they illustrate how such quantum space-times might look like,and provide a possible explanation of the $\mathrm{BB}$, beyond postulating that it arises from random quantum fluctuations as in other approaches [20]. The BB here is simply a feature of the emergent geometry, which is extended by a Euclidean regime. It arises in the presence of different space-like and time-like masses $m^{2} \neq m_{0}^{2}$ in the matrix model action satisfying certain conditions. Even though the solutions may not be realistic and stability at the

\footnotetext{
${ }^{1}$ Note that the branes should be viewed as classical condensates here, this is not a holographic scenario, and it does not rely on quantum effects.

${ }^{2}$ The effective metric can be thought of as open string metric in the Seiberg-Witten limit [11].
} 
quantum level is not established, they nicely illustrate the appeal and the scope of the IKKT model (or similar matrix models) as a fundamental theory of space-time and matter.

\section{Lorentzian matrix models}

We are interested in solutions of the following IKKT-type matrix model [1] with mass terms

$$
S[Y, \Psi]=\frac{1}{g^{2}} \operatorname{Tr}\left(\left[Y^{a}, Y^{b}\right]\left[Y^{a^{\prime}}, Y^{b^{\prime}}\right] \eta_{a a^{\prime}} \eta_{b b^{\prime}}-m^{2} Y^{i} Y^{i}+m_{0}^{2} Y^{0} Y^{0}+\bar{\Psi} \Gamma_{a}\left[Y^{a}, \Psi\right]\right) .
$$

Here $\eta_{a b}=\operatorname{diag}(-1,1, \ldots, 1)$ is interpreted as Minkowski metric of the target space $\mathbb{R}^{1, D-1}$. Indices $i$ indicate Euclidean directions, and 0 is the time-like direction. Fermions $\Psi$ are included via the Gamma matrices $\Gamma^{a}$ to enable supersymmetry, however we will focus on the bosonic sector from now on. The above model leads to the classical equations of motion

$$
\begin{aligned}
-\square_{Y} Y^{i}-m^{2} Y^{i} & =0 \\
\square_{Y} Y^{0}+m_{0}^{2} Y^{0} & =0
\end{aligned}
$$

where

$$
\square_{Y}=\eta_{a b}\left[Y^{a},\left[Y^{b}, .\right]\right]
$$

plays the role of the d'Alembertian. We will study solutions of these equations which are interpreted as 3+1-dimensional space-times, more specifically as noncommutative "branes" embedded in target space.

As emphasized in the introduction, the choice of the matrix model is important. The picture of classical brane solutions $\mathcal{M}$ is presumably justified only for the maximally supersymmetric IKKT model with $D=9+1$ [1], due to UV cancellations of the quantum fluctuations; in fact this model reduces to $\mathcal{N}=4$ SYM on 4-dimensional backgrounds. ${ }^{3}$ Thus although the solutions given below are not supersymmetric, the underlying model (2.1) is, up to the soft mass terms. Hence SUSY is broken spontaneously and softly, but we expect that this still ensures sufficient UV cancellations to tame the quantum corrections.

These mass terms are important because they introduce a scale into the model, and conversely quantum corrections are expected to induce such mass terms on curved backgrounds. Indeed as discussed in [15-17], after taking into account an IR cutoff and integrating out the scale factor in the matrix path integral

$$
Z=\int d Y d \psi e^{i S_{\mathrm{IKKT}}[Y, \psi]}
$$

the equations of motions (2.2) arise, with $m^{2} \neq m_{0}^{2}$ resulting from an IR regularization which mildly breaks Lorentz invariance. Since we only study classical solutions of (2.2) and their geometrical properties, we will restrict ourselves to the bosonic part of (2.1), including the mass terms by hand. Moreover, we will see that a Big Bang arises from the present solutions only if $m^{2} \neq m_{0}^{2}$. In fact there are no finite-dimensional non-trivial solutions without a mass term, as shown in the appendix.

\footnotetext{
${ }^{3}$ This is the only model of a noncommutative gauge theory where quantum corrections are tame and expected to be perturbatively finite [21].
} 


\section{$3 \quad$ Recollapsing universe from fuzzy 4-spheres}

\subsection{The Euclidean fuzzy 4-sphere}

We briefly recall the definition of fuzzy 4 -spheres [22], cf. [23-27]. The starting point is the Lie algebra $\mathfrak{s o}(6) \cong \mathfrak{s u}(4)$, with generators $\mathcal{M}^{a b}, a, b=1, \ldots, 6$ and commutation relations

$$
\left[\mathcal{M}_{a b}, \mathcal{M}_{c d}\right]=i\left(\delta_{a c} \mathcal{M}_{b d}-\delta_{a d} \mathcal{M}_{b c}-\delta_{b c} \mathcal{M}_{a d}+\delta_{b d} \mathcal{M}_{a c}\right) .
$$

Now consider the embedding of $\mathrm{SO}(5) \subset \mathrm{SO}(6)$ defined by restricting the indices of $\mathcal{M}^{a b}$ to be in $\{1, \ldots, 5\}$, and denote the remaining generators as

$$
\begin{aligned}
X^{a} & =r \mathcal{M}^{a 6}, \quad a=1, \ldots, 5, \\
{\left[X^{a}, X^{b}\right] } & =\Theta^{a b}=r^{2} \mathcal{M}^{a b}
\end{aligned}
$$

Here $r$ is a scale with dimension length. By construction, the $X^{a}$ transform covariantly under $\mathrm{SO}(5)$ generated by $\mathcal{M}^{a b}$,

$$
\left[\mathcal{M}^{a b}, X^{c}\right]=i\left(g^{a c} X^{b}-g^{b c} X^{a}\right),
$$

We fix the $\mathrm{SO}(6)$ representation to be $\mathcal{H}=(0,0, N)=\left(\mathbb{C}^{4}\right)^{\otimes_{S} N}$, which is well-known to remain irreducible under $\mathrm{SO}(5)$. Therefore the radius is a constant,

$$
X^{a} X_{a}=X^{a} X^{b} \delta_{a b}=R^{2} \mathbb{1}=r^{2} R_{N}^{2} \mathbb{1}, \quad R_{N}^{2}=\frac{1}{4} N(N+4) .
$$

The $\mathfrak{s o}(6) \cong \mathfrak{s u}(4)$ generators $\mathcal{M}^{a b} \in \operatorname{End}(\mathcal{H}), a, b=1, \ldots, 6$ are now understood as quantized embedding functions

$$
\mathcal{M}^{a b} \sim m^{a b}: \quad \mathbb{C} P^{3} \hookrightarrow \mathfrak{s o}(6) \cong \mathbb{R}^{15}
$$

where $m^{a b}=r^{-2} \theta^{a b}$, and similarly

$$
X^{a} \sim x^{a}: \quad \mathbb{C} P^{3} \hookrightarrow \mathbb{R}^{5} .
$$

In the semi-classical limit, the commutators reduce to the Poisson bracket on $\mathbb{C} P^{3}$, and we can work with the Poisson structure

$$
\left\{x^{a}, x^{b}\right\}=i \theta^{a b} .
$$

Therefore the semi-classical geometry underlying fuzzy $S_{N}^{4}$ is $\mathbb{C} P^{3}$, which is an $S^{2}$ - bundle over $S^{4}$ carrying a canonical symplectic structure, and $x^{a}: \mathbb{C} P^{3} \rightarrow S^{4} \subset \mathbb{R}^{5}$ is nothing but the Hopf map. This can also be justified e.g. via coherent states $|x, \xi\rangle$, which are in one-to-one correspondence (up to a phase) to points on $\mathbb{C} P^{3} \cong \mathrm{SU}(4) / \mathrm{SU}(3) \times \mathrm{U}(1)$, which is locally isomorphic to $S^{4} \times S^{2} \ni(x, \xi)$. It turns out that $\theta^{a b}=\theta^{a b}(x, \xi)$ is tangential $x_{a} \theta^{a b}=0$ on $S^{4}$, and transforms under the local stabilizer $\mathrm{SO}(4)_{x}$ of any point $x \in S^{4}$. More precisely, it forms a bundle of self-dual bi-vectors $\theta^{\mu \nu}$ on $S^{4}$, which is locally isomorphic to $S^{4} \times S^{2}$. In particular, $\left[\theta^{a b}\right]_{S^{2}}=0$ where $[.]_{S^{2}}$ denotes the averaging over the internal $S^{2}$. For more details on fuzzy $S_{N}^{4}$ we refer to [27-29]. A gentle introduction to the geometrical concepts of fuzzy spaces can be found e.g. in [30]. 


\subsection{Lorentzian fuzzy 4 -sphere in $\mathbb{R}^{1,4}$}

We will show that ellipsoidal deformations of $S_{N}^{4}$ are exact solutions ${ }^{4}$ of the model (2.1), provided $m^{2} \neq m_{0}^{2}$. Thus let $\mathcal{M}^{a b}$ be hermitian generators of an irrep of $\mathrm{SO}(6)$ as above which remains irreducible under $\mathrm{SO}(5)$. Define $Y^{a}, a \in\{0, \ldots, 4\}$ by

$$
Y^{i}=X^{i}, \quad \text { for } i=1, \ldots, 4, \quad Y^{0}=\kappa X^{5}
$$

for $X^{a}=r \mathcal{M}^{a 6}$ as in (3.2). Clearly the $Y^{i}, i=1, \ldots, 4$ transform as vectors under $\mathrm{SO}(4) \subset$ $\mathrm{SO}(5)$. We ask these $Y^{a}$ to be solutions of the mass-deformed matrix model (2.1), which in terms of the $X^{a}$ variables looks as follows

$$
S=\frac{1}{g^{2}} \operatorname{Tr}\left(\left[X^{a}, X^{b}\right]\left[X^{a^{\prime}}, X^{b^{\prime}}\right] g_{a a^{\prime}} g_{b b^{\prime}}-m^{2} X^{i} X^{i}+\tilde{m}_{0}^{2} X^{0} X^{0}\right),
$$

Now the target space metric in these coordinates is

$$
g_{a b}=\operatorname{diag}\left(-\kappa^{2}, 1,1,1,1\right), \quad \text { and } \quad \tilde{\mathrm{m}}_{0}^{2}=\kappa^{2} \mathrm{~m}_{0}^{2} .
$$

The commutation relations (3.2) give

$$
\begin{aligned}
{\left[X^{a},\left[X^{a}, X^{b}\right]\right] } & =i r^{2}\left[X^{a}, \mathcal{M}^{a b}\right]=i r^{2}\left[\mathcal{M}^{b a}, X^{a}\right] \quad \text { (no sum) } \\
& =r^{2}\left\{\begin{array}{ll}
X^{b}, & b \neq a \\
0, & b=a
\end{array} .\right.
\end{aligned}
$$

Hence the equations of motion

$$
\left(\square_{X}+\tilde{m}_{0}^{2}\right) X^{0}=0=\left(\square_{X}+m^{2}\right) X^{i}
$$

imply

$$
4 r^{2}+\tilde{m}_{0}^{2}=0=\left(3-\kappa^{2}\right) r^{2}+m^{2} .
$$

This clearly requires $m_{0}^{2}<0$, and

$$
\frac{3-\kappa^{2}}{4}=\frac{m^{2}}{\tilde{m}_{0}^{2}}=\frac{m^{2}}{\kappa^{2} m_{0}^{2}} .
$$

Hence for any $m_{0}^{2}<0 \leq m^{2}$ in the original model (2.1), there is a unique

$$
\kappa^{2} \geq 3, \quad \text { or } \kappa^{2}=3 \text { for } m=0
$$

and $r^{2}>0$ so that $Y^{a}$ is a solution of (2.1), or equivalently $S_{N}^{4}$ with $X^{a} X^{b} \delta_{a b}=R^{2}(3.4)$ is a solution of the matrix model with Lorentzian target space metric $g_{a b}$ (3.10). There are also $S_{N}^{4}$ solutions for $m_{0}^{2}<m^{2}<0$ as long as $\frac{m^{2}}{m_{0}^{2}} \leq \frac{9}{16}$, but we will see that they do not

\footnotetext{
${ }^{4}$ It is well-known that fuzzy $S_{N}^{4}$ is a solution upon including a quintic term $\operatorname{Tr}(\varepsilon Y Y Y Y Y)$ [26]. However this is not a soft term, and thus quantum effects are problematic [31]. Here we show that such a term is not necessary in the presence of a mass term. The 4-dimensional cosmologies in [13] are not fully covariant but carry Poisson-structures which break $\mathrm{SO}(4)$ invariance. This is avoided here.
} 
acquire a Minkowski metric. However for $m^{2}<m_{0}^{2}<0$, we will find expanding universe solutions with Minkowski metric, which are discussed in section 4 .

To study the geometry in more detail, we restrict ourselves to the semi-classical limit from now on, replacing commutators by Poisson brackets as discussed in section 3.1. Then (3.6) is replaced by $X^{a} \sim x^{a}: \mathbb{C} P^{3} \hookrightarrow S^{4} \subset \mathbb{R}^{1,4}$. Hence the image of $\mathbb{C} P^{3}$ in $\mathbb{R}^{1,4}$ defines a manifold $\mathcal{M}$ which is topologically a 4 -sphere carrying a bundle of bivectors $\theta^{\mu \nu}$ (which are self-dual w.r.t. its Euclidean $\mathrm{SO}(5)$-invariant metric), but embedded in Lorentzian target space $\mathbb{R}^{1,4}$. All these structures will play a role, and one must be careful to use them appropriately.

We are particularly interested in the metric on $\mathcal{M}$. There are in fact two different metrics on the brane $\mathcal{M} \subset \mathbb{R}^{1,4}$, as in string theory: the induced metric is simply the pullback of the constant ("closed string ") metric in target space $\mathbb{R}^{1,4}$, and it will be determined first. This is distinct from the effective metric, which governs the (noncommutative) gauge theory on the brane $\mathcal{M}$, which arises from fluctuations ${ }^{5} X^{a} \rightarrow X^{a}+\mathcal{A}^{a}$ in the matrix model. This is the analog of the open string metric [11], and it will be determined in a second step. For a more general discussion of these topics see e.g. [9].

Induced metric. As a warm-up, we compute the induced metric $g_{\mu \nu}$ on $\mathcal{M} \subset \mathbb{R}^{1,4}$. This clearly has Euclidean signature at $x_{0}= \pm R$, and Minkowski signature for $x_{0} \approx 0$. The domains of fixed signature are separated by a space-like $S^{3} \subset S^{4}$ where the metric is degenerate. This is the locus on $S^{4}$ where the tangent space includes a null direction of $\mathbb{R}^{4,1}$. Using the space-like $\mathrm{SO}(4)$ symmetry, we can choose a standard reference point

$$
x=\left(x_{0}, x_{1}, 0,0,0\right)=R(\cos (\eta), \sin (\eta), 0,0,0) \in S^{4} \subset \mathbb{R}^{4,1}, \quad x_{0}^{2}+x_{1}^{2}=R^{2}
$$

and use the tangential coordinate

$$
\tau=R \eta
$$

which points in the $x^{0} x^{1}$ direction,

$$
\frac{d}{d \tau} x=R(-\sin (\eta), \cos (\eta), 0,0,0)
$$

Then the induced metric is

$$
g_{\mu \nu}=\operatorname{diag}\left(\cos ^{2}(\eta)-\kappa^{2} \sin ^{2}(\eta), 1,1,1\right)
$$

in local $x^{\mu}=\left(\tau x^{2} x^{3} x^{4}\right)$ coordinates on $T_{p} \mathcal{M}$ at the standard reference point (3.16), or

$$
\begin{aligned}
d s_{g}^{2} & =R^{2}\left(\cos ^{2}(\eta)-\kappa^{2} \sin ^{2}(\eta)\right) d \eta^{2}+R^{2} \sin ^{2}(\eta) d \Omega_{3}^{2} \\
& =\beta^{2}(\eta) d \eta^{2}+\alpha^{2}(\eta) d \Omega_{3}^{2}
\end{aligned}
$$

in FRW coordinates where $d \Omega_{3}^{2}$ is the $\mathrm{SO}(3)$-invariant metric on the unit sphere $S^{3}$ and

$$
\beta^{2}(\eta)=\frac{1}{2} R^{2}\left(\left(\kappa^{2}+1\right) \cos (2 \eta)-\left(\kappa^{2}-1\right)\right), \quad \alpha^{2}=\frac{1}{2} R^{2}(1-\cos (2 \eta)) .
$$

\footnotetext{
${ }^{5}$ Note that $\mathcal{A}^{a} \in \operatorname{End}(\mathcal{H}) \cong \mathcal{C}\left(\mathbb{C} P^{3}\right)$ describes indeed functions living on $\mathcal{M}$.
} 
Clearly $\alpha \geq 0$ vanishes only on the poles $x^{0}= \pm R$ where $\eta=0, \pi$. In contrast, $\beta\left(\eta_{*}\right)=0$ vanishes if

$$
\cos \left(2 \eta_{*}\right)=\frac{\kappa^{2}-1}{\kappa^{2}+1}
$$

which is $\eta_{*}=\frac{\pi}{6}$ for $\kappa^{2}=3$. Hence there is indeed an interesting transition from Euclidean to Minkowski signature, however the associated singularity cannot be interpreted as Big Bang, as there is no rapid initial expansion. Therefore the induced metric does not give rise to an interesting cosmology. In contrast, we will see that a Big Bang does arise for the effective metric. The volume-form arising from the 4 -form flux will be crucial for this mechanism.

Effective metric and averaging. We now compute the effective metric on $\mathcal{M} \subset \mathbb{R}^{1,4}$ in the matrix model. It is easiest to use the $x^{a}$ description where the embedding is spherical, but the target space metric is $g_{a b}$ (3.10). The effective metric $G^{\mu \nu}$ in matrix models is determined by the kinetic term for a scalar field ${ }^{6}$ as follows $[9,10,30]$

$$
\begin{aligned}
S[\phi] & =-\operatorname{Tr}\left[X^{a}, \phi\right]\left[X^{b}, \phi\right] g_{a b} \sim \frac{\operatorname{dim} \mathcal{H}}{\operatorname{Vol}_{\omega}(\mathcal{M})} \int_{\mathcal{M}} d^{4} x \sqrt{\left|\theta^{\mu \nu}\right|^{-1}} \gamma^{\mu \nu} \partial_{\mu} \phi \partial_{\nu} \phi \\
& =\int_{\mathcal{M}} d^{4} x \sqrt{\left|G_{\mu \nu}\right|} G^{\mu \nu} \partial_{\mu} \varphi \partial_{\nu} \varphi .
\end{aligned}
$$

using greek indices for local coordinates on $\mathcal{M}=S^{4}$. Here $\varphi=c \phi$ has dimension mass, $\sqrt{\left|\theta^{\mu \nu}\right|^{-1}}$ is the $\mathrm{SO}(5)$-invariant Euclidean volume form on $S^{4} \subset \mathbb{R}^{5}$ inherited from the symplectic form $\omega$ on $\mathbb{C} P^{3} \sim S^{4} \times S^{2}$, and

$$
\gamma^{\mu \nu}=g_{\mu^{\prime} \nu^{\prime}}\left[\theta^{\mu^{\prime} \mu} \theta^{\nu^{\prime} \nu}\right]_{S^{2}} .
$$

This is reminiscent of the open string metric in the Seiberg-Witten limit [11]. The crucial volume-form arises because $\operatorname{Tr} \sim \int_{\mathbb{C} P^{3}} \omega^{\wedge 3} \sim \int_{S^{4}} d^{4} x \sqrt{\left|\theta^{\mu \nu}\right|^{-1}}$ is an integral over the symplectic manifold $\mathbb{C} P^{3}$. Since $\left|\theta^{\mu \nu}\right|$ is constant along the internal $S^{2}$ fiber over $S^{4}$, the $S^{2}$ only contributes an irrelevant constant factor which is dropped. Assuming that lowenergy fields $\phi(x)$ are constant along $S^{2},(3.24)$ follows. Recasting this kinetic term in the standard covariant metric form, we can read off the conformal factor ${ }^{7}$

$$
G^{\mu \nu}=\alpha \gamma^{\mu \nu}, \quad \alpha=\sqrt{\frac{\left|\theta^{\mu \nu}\right|}{\left|\gamma^{\mu \nu}\right|}} .
$$

The average $\left[\theta^{\mu^{\prime} \mu} \theta^{\nu^{\prime} \nu}\right]_{S^{2}}$ can be evaluated using the $S_{N}^{4}$ formula [10]

$$
\left[\theta^{a b} \theta^{c d}\right]_{S^{2}}=\frac{1}{12} \Delta^{4}\left(P_{S}^{a c} P_{S}^{b d}-P_{S}^{b c} P_{S}^{a d}+\varepsilon^{a b c d e} \frac{1}{R} x^{e}\right)
$$

where $\Delta^{2}=2 r R$ is the space-time uncertainty scale, and

$$
P_{S}^{a c}(x)=\delta^{a c}-\frac{1}{R^{2}} x^{a} x^{c}, \quad R^{2}=\delta_{a b} x^{a} x^{b}
$$

\footnotetext{
${ }^{6}$ The conformal factor cannot be determined from gauge fields because of conformal invariance.

${ }^{7}$ The formula given in [9] is modified here due to the averaging over $S^{2}$.
} 
is the Euclidean (!) projector $P_{S}^{a b} \delta_{b c} P_{S}^{c d}=P_{S}^{a d}$ on the tangent space of $S^{4}$. Note that (3.26) is the unique $\mathrm{SO}(5)$ - invariant tensor which reflects the antisymmetry and selfduality ${ }^{8}$ of $\theta^{a b}$. Then

$$
\begin{aligned}
g_{a b} P_{S}^{a b} & =\left(-\kappa^{2}+4\right)-\frac{1}{R^{2}} g_{a c} x^{a} x^{c}=\left(-\kappa^{2}+4\right)+\frac{1}{R^{2}}\left(\kappa^{2} x_{0}^{2}-\left(R^{2}-x_{0}^{2}\right)\right) \\
& =\left(-\kappa^{2}+3\right)+\left(\kappa^{2}+1\right) \cos ^{2}(\eta)
\end{aligned}
$$

so that

$$
\begin{aligned}
\gamma^{b d} & =\frac{1}{12} \Delta^{4}\left(g_{a b} P_{S}^{a b} P_{S}^{b d}-g_{a c} P_{S}^{b c} P_{S}^{a d}\right) \\
& =: \frac{1}{12} \Delta^{4} \tilde{\gamma}_{a c} P_{S}^{b c} P_{S}^{a d}
\end{aligned}
$$

with

$$
\tilde{\gamma}_{a c}=\left(\left(1+\kappa^{2}\right) \cos ^{2}(\eta)+\left(3-\kappa^{2}\right)\right) P_{a c}^{S}-g_{a c} .
$$

Before continuing with the evaluation, we consider some special cases. On the maximal space-like $S^{3}$ with $x^{0}=0$, the first term vanishes, and

$$
\tilde{\gamma}_{a b}=-\eta_{a b}=\operatorname{diag}\left(\kappa^{2},-1-1,-1,-1\right), \quad \mathrm{x}^{0}=0 .
$$

This is indeed Lorentzian, as desired. In contrast, for $x_{0}= \pm R_{x}$ we obtain

$$
\begin{aligned}
\tilde{\gamma}_{a c} & =\frac{1}{12} \Delta^{4}\left(4 P_{a c}^{S}-\eta_{a c}\right)=\frac{1}{12} \Delta^{4} \operatorname{diag}\left((0,4,4,4,4)-\left(-\kappa^{2}, 1,1,1,1\right)\right) \\
\kappa^{2} & =3 \frac{1}{12} \Delta^{4} \operatorname{diag}(3,3,3,3,3)
\end{aligned}
$$

which is Euclidean. Hence the space-like $\tilde{\gamma}_{i i}$ vanish somewhere in between; therefore there must be some singularities, which are tentatively interpreted as Big Bang and Big Crunch. In contrast, the $\tilde{\gamma}_{00}$ component never vanishes.

Now we determine the effective metric explicitly. We use the local $x^{\mu}=\left(\tau x^{2} x^{3} x^{4}\right)$ coordinates on $T_{p} \mathcal{M}$ at the standard reference point $(3.16)$, where $P_{S}=\operatorname{diag}(1,1,1,1)$. Then

$$
\begin{aligned}
\tilde{\gamma}_{i i} & =\left(\kappa^{2}+1\right) \cos ^{2}(\eta)+2-\kappa^{2}=\frac{1}{2}\left(\left(\kappa^{2}+1\right) \cos (2 \eta)+\left(-\kappa^{2}+5\right)\right) \\
& =: 3 c(\eta), \quad i=2,3,4 \\
\tilde{\gamma}_{\tau \tau} & =\left(\left(1+\kappa^{2}\right) \cos ^{2}(\eta)+\left(3-\kappa^{2}\right)\right) P_{\tau \tau}-g_{\tau \tau} \\
& =3
\end{aligned}
$$

using

$$
\begin{aligned}
g_{\tau \tau} & =(-\sin \eta, \cos \eta) \operatorname{diag}\left(-\kappa^{2}, 1\right)(-\sin \eta, \cos \eta) \\
& =\left(\kappa^{2}+1\right) \cos ^{2} \eta-\kappa^{2} .
\end{aligned}
$$

\footnotetext{
${ }^{8}$ One must be careful not to mix up the Euclidean and Lorentzian aspects. Selfduality of course holds w.r.t. the Euclidean metric. In the same vein, the trace in (3.23) has nothing to do with the target space metric, and it reduces to the integral over the symplectic $\mathbb{C} P^{3} \sim S^{4} \times S^{2}$ as in the Euclidean case.
} 


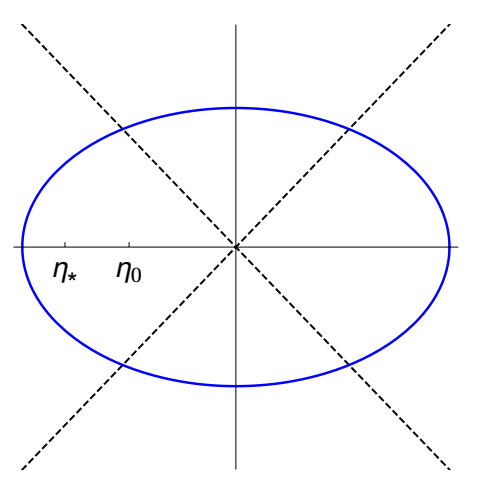

Figure 1. Schematic picture of the recollapsing universe with lightcone for $g_{\mu \nu}$, indicating $\eta_{*}$ and $\eta_{0}$.

Therefore

$$
\gamma^{\mu \nu}=\frac{1}{4} \Delta^{4} \operatorname{diag}(1, \mathrm{c}(\eta), \mathrm{c}(\eta), \mathrm{c}(\eta))
$$

consistent with the cases $x_{0}= \pm R_{x}$ and $x_{0}=0$ since $c(0)=1$.

A singularity occurs if the space-components $\tilde{\gamma}_{i i}$ change sign, i.e. for $c\left(\eta_{0}\right)=0$. This happens for

$$
\cos \left(2 \eta_{0}\right)=\frac{\kappa^{2}-5}{\kappa^{2}+1}
$$

and is interpreted as Big Bang and Big Crunch. This always has a solution since $\kappa^{2} \geq$ 3 (3.15), which occurs always after the signature change (3.22) for the induced metric, $\eta_{0}>\eta_{*}$ (in the expanding phase), as indicated in figure 1. For $\kappa^{2}=3$, this occurs for $\eta_{0}=\frac{\pi}{3}$. Between Big Bang and Big Crunch, $\gamma^{\mu \nu}$ has signature ${ }^{9}(+---)$ since $c(\eta)<0$.

In the same coordinates, the $\mathrm{SO}(5)$-invariant volume form $\sqrt{\left|\theta^{\mu \nu}\right|} \sim \frac{\Delta^{4}}{4}$ is constant. Therefore the conformal factor (3.25) is

$$
\alpha=\sqrt{\frac{\left|\theta^{\mu \nu}\right|}{\left|\gamma^{\mu \nu}\right|}}=\frac{4}{\Delta^{4}}|c(\eta)|^{-3 / 2},
$$

and we obtain the effective metric

$$
\begin{aligned}
& G^{\mu \nu}=|c(\eta)|^{-3 / 2} \operatorname{diag}(1, \mathrm{c}(\eta), \mathrm{c}(\eta), \mathrm{c}(\eta)) \\
& G_{\mu \nu}=|c(\eta)|^{3 / 2} \operatorname{diag}\left(1, \mathrm{c}(\eta)^{-1}, \mathrm{c}(\eta)^{-1}, \mathrm{c}(\eta)^{-1}\right) .
\end{aligned}
$$

Scale factor. To extract the cosmological evolution, we express this metric in FRW coordinates,

$$
d s_{G}^{2}=b^{2}(\eta) d \eta^{2}-\tilde{a}^{2}(\eta) d \Omega^{2}=d t^{2}-a^{2}(t) d \Omega^{2}
$$

\footnotetext{
${ }^{9}$ Note that the effective metric has the opposite sign of the induced metric. This is due to the Poisson structure which enters $\gamma^{a b}$.
} 
where $d \Omega^{2}$ is the length element on a spatial 3 -sphere $S^{3}$ with unit radius. Thus

$$
\tilde{a}^{2}(\eta)=R^{2}|c(\eta)|^{1 / 2}=a^{2}(t), \quad b^{2}(\eta)=R^{2}|c(\eta)|^{3 / 2}
$$

in the cosmological era (with Minkowski signature). Note that now both $a$ and $b$ vanish at the time $\eta_{0}$ of the $\mathrm{BB}$, in contrast to the induced metric (3.19). We set $R=1$ for simplicity. Then

$$
b=a^{3}
$$

The comoving time parameter $t$ is determined as

$$
\dot{\eta}=b^{-1}=a^{-3} .
$$

We can solve this in the cosmological era recalling (3.36),

$$
a^{4}=|c(\eta)|=\frac{1}{6}\left(\left(\kappa^{2}-5\right)-\left(\kappa^{2}+1\right) \cos (2 \eta)\right),
$$

which gives

$$
4 a^{3} \dot{a}=\frac{1}{3}\left(\kappa^{2}+1\right) \sin (2 \eta) \dot{\eta}=\frac{1}{3}\left(\kappa^{2}+1\right) a^{-3} \sqrt{1-\frac{\left(6 a^{4}-\kappa^{2}+5\right)^{2}}{\left(\kappa^{2}+1\right)^{2}}}
$$

and finally

$$
\dot{a}=\frac{1}{12} a^{-6} \sqrt{\left(\kappa^{2}+1\right)^{2}-\left(6 a^{4}-\kappa^{2}+5\right)^{2}} .
$$

At early times after the BB, this is approximated by

$$
\dot{a}=c a^{-6}, \quad c=\frac{\sqrt{\kappa^{2}-2}}{2 \sqrt{3}}
$$

which leads to the initial expansion

$$
a(t) \sim t^{1 / 7}
$$

Hence the scale parameter $a(t)$ exhibits a very rapid (but not exponential) initial expansion, which slows down naturally. It reaches a maximum $a_{\max }$ at

$$
a_{\max }^{4}=\frac{\kappa^{2}-2}{3} \geq \frac{1}{3}
$$

after which the universe starts to contract, and eventually collapses in a Big Crunch. It is decelerating at all times, $\ddot{a}<0$. The Hubble parameter is

$$
\begin{aligned}
H(t)=\frac{\dot{a}}{a} & =\frac{1}{12} a^{-7} \sqrt{\left(\kappa^{2}+1\right)^{2}-\left(6 a^{4}-\kappa^{2}+5\right)^{2}} \\
& \sim t^{-1}, \quad t \approx 0
\end{aligned}
$$

for the early universe. 


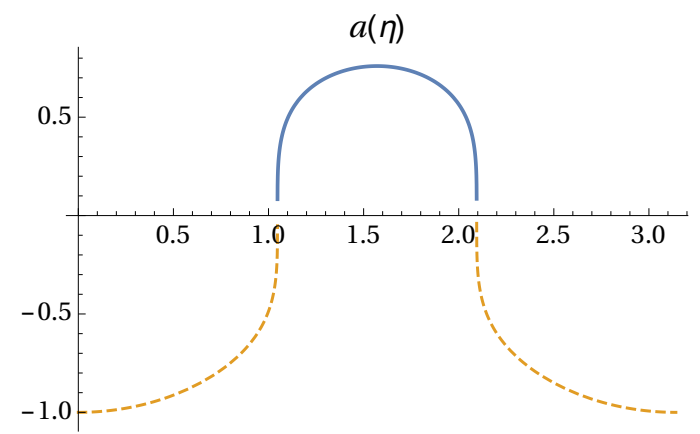

Figure 2. $a(\eta)$ for the $S^{4}$ cosmology with $\kappa^{2}=3$. The red dashed line describes the Euclidean caps with imaginary $a(\eta)$.

To see what this means from the target space point of view, we plot $a(\eta)$ in figure 2 as a function of the target space angle $\eta$, rather than $a(t)$. Then the initial singularity is milder than in the comoving time $t$, but still manifest. Note that the scale parameter $a(\eta)$ is imaginary for $0 \leq \eta<\eta_{0}$, as indicated in figure 2. This gives the Euclidean effective metric for $\eta<\eta_{0}$. Since the BB (3.36) occurs after the signature change in the induced metric (3.22) at $\eta_{*}$, there is an era before the BB where the effective metric is Euclidean but the induced metric is Lorentzian. The induced metric governs the one-loop corrections, which in the IKKT model essentially gives IIB supergravity in the 10D bulk, i.e. the closed string sector with short-range $r^{-8}$ propagators [1-4]. This should entail some causal connection even before the $\mathrm{BB}$ (due to the closed string sector), which might resolve the horizon problem even in the absence of standard inflation, and thereby explain the observed uniformity in the CMB.

It is instructive to compare the effective metric $G_{\mu \nu}$ (3.38) with the induced metric $g_{\mu \nu}$ (3.19). The crucial difference lies in the conformal factor, which is responsible for the expanding BB behavior for $G_{\mu \nu}$ rather than just developing a $(0+++)$ degeneracy for $g_{\mu \nu}$. We emphasize again that this conformal factor arises from matching the kinetic term in the matrix model action with a covariant metric expression. Since the matrix model action involves the trace, it incorporates a measure (a density) which arises from the underlying symplectic manifold $\mathbb{C} P^{3}$, corresponding to a quantized 4-form flux on $S^{4}$.

\section{Expanding universe from fuzzy hyperboloids}

Now we repeat the above computation for the case of a hyperboloid. We focus on the fuzzy hyperboloid $H_{n}^{4}$ as discussed in $[32,33]$. Analogous to $S_{N}^{4}$, this arises from certain irreducible representations of the noncompact cousin $\mathrm{SO}(1,4)$ of $\mathrm{SO}(5)$, and again there are magic representations where this structure group is enhanced to $\mathrm{SO}(2,4)$.

\subsection{Euclidean fuzzy hyperboloids}

To define fuzzy $H_{n}^{4}$, let $\eta^{a b}=\operatorname{diag}(-1,1,1,1,1,-1)$ be the invariant metric of $\operatorname{SO}(4,2)$, and $\mathcal{M}^{a b}$ be hermitian generators of $\mathrm{SO}(4,2)$, which satisfy

$$
\left[\mathcal{M}_{a b}, \mathcal{M}_{c d}\right]=i\left(\eta_{a c} \mathcal{M}_{b d}-\eta_{a d} \mathcal{M}_{b c}-\eta_{b c} \mathcal{M}_{a d}+\eta_{b d} \mathcal{M}_{a c}\right)
$$


We choose a particular type of (massless discrete series) positive-energy unitary irreps ${ }^{10}$ $\mathcal{H}_{n}$ known as "minireps" or doubletons [34-36], which have the remarkable property that they remain irreducible ${ }^{11}$ under $\mathrm{SO}(4,1) \subset \mathrm{SO}(4,2)$. They have positive discrete spectrum

$$
\operatorname{spec}\left(\mathcal{M}^{05}\right)=\left\{E_{0}, E_{0}+1, \ldots\right\}, \quad E_{0}=1+\frac{n}{2}
$$

where the eigenspace with lowest eigenvalue of $\mathcal{M}^{05}$ is an $n+1$-dimensional irreducible representation of either $S U(2)_{L}$ or $S U(2)_{R}$. Then the hermitian generators

$$
\begin{aligned}
X^{a} & :=r \mathcal{M}^{a 5}, \quad a=0, \ldots, 4 \\
{\left[X^{a}, X^{b}\right] } & =i r^{2} \mathcal{M}^{a b}=: i \Theta^{a b}
\end{aligned}
$$

satisfy

$$
\eta_{a b} X^{a} X^{b}=X^{i} X^{i}-X^{0} X^{0}=-R^{2} \mathbb{1}
$$

with $R^{2}=r^{2}\left(n^{2}-4\right)$ [32]. Since $X^{0}=r \mathcal{M}^{05}>0$ has positive spectrum, this describes a one-sided hyperboloid in $\mathbb{R}^{1,4}$, denoted as $H_{n}^{4}$. Analogous to fuzzy $S_{N}^{4}$, the semi-classical geometry underlying $H_{n}^{4}$ is $\mathbb{C} P^{1,2}$ [32], which is an $S^{2}$ - bundle over $H^{4}$ carrying a canonical symplectic structure. In the fuzzy case, this fiber is a fuzzy 2-sphere $S_{n}^{2}$. We work again in the semi-classical limit. It is important to note that the induced metric on the hyperboloid $\mathcal{M}:=H^{4} \subset \mathbb{R}^{1,4}$ is Euclidean, despite the $\mathrm{SO}(4,1)$ isometry. This is obvious at the point $x=(R, 0,0,0,0)$, where the tangent space is $\mathbb{R}_{1234}^{4}$.

Thus $H_{n}^{4}$ has the same local structure as $S_{n}^{4}$ in the semi-classical limit, with a Poisson tensor $\theta^{\mu \nu}(x, \xi)$ transforming as a 2-form under the local stabilizer $\mathrm{SO}(4)_{x}$ of any point $x \in \mathcal{M}$. This realizes a $S^{2}$ bundle of self-dual 2-vectors. Then the averaging over $S^{2}$ can be achieved using the same local formulas as for $S_{N}^{4}$, which will be useful below.

In particular, $H_{n}^{4}$ has a finite density of microstates just like $S_{n}^{4}$, since the number of states in $\mathcal{H}_{n}$ between two given $X^{0}$-eigenvalues is finite. This density can in fact be much smaller than for $S_{N}^{4}$, because $n$ is no longer required to be large as we will see.

\subsection{Lorentzian fuzzy hyperboloids}

In analogy to (3.8), Lorentzian spaces of the above type with suitable rescaling of the generators $X^{a}$ are solutions of the same matrix model (2.1), for suitable mass parameters. Thus we look for solutions of the mass-deformed matrix model given by rescaled generators $Y^{a}, a \in\{0, \ldots, 4\}$

$$
Y^{i}=X^{i}, \quad \text { for } i=1, \ldots, 4, \quad Y^{0}=\kappa X^{5} .
$$

The transform as vectors of $\mathrm{SO}(4)$. We can again rewrite the model in the $X^{a}$ coordinates as in (3.9), with

$$
g_{a b}=\operatorname{diag}\left(-\kappa^{2}, 1,1,1,1\right) .
$$

\footnotetext{
${ }^{10}$ Strictly speaking there are two versions $\mathcal{H}_{n}^{L}$ or $\mathcal{H}_{n}^{R}$ with opposite "chirality", but this distinction is irrelevant in the present paper and therefore dropped.

${ }^{11}$ This follows from the minimal oscillator construction of $\mathcal{H}_{n}$, where all $\mathrm{SO}(4,2)$ weight multiplicities are at most one. Cf. [36-38].
} 
The commutation relations now give

$$
\begin{aligned}
{\left[X^{a},\left[X^{a}, X^{b}\right]\right] } & =i r^{2}\left[X^{a}, \mathcal{M}^{a b}\right]=i r^{2}\left[\mathcal{M}^{b a}, X^{a}\right] \quad \text { (no sum) } \\
& =r^{2} \begin{cases}X^{b}, & b \neq a \neq 0 \\
-X^{b}, & b \neq a=0 \\
0, & b=a\end{cases}
\end{aligned}
$$

Thus the equations of motion

$$
\left(\square_{X}+\tilde{m}_{0}^{2}\right) X^{0}=0=\left(\square_{X}+m^{2}\right) X^{i}
$$

reduce to

$$
4 r^{2}+\tilde{m}_{0}^{2}=0=\left(3+\kappa^{2}\right) r^{2}+m^{2} .
$$

Now both mass terms need to be negative $m_{0}^{2}<0$ and $m^{2}<0$, with

$$
\frac{3+\kappa^{2}}{4}=\frac{m^{2}}{\tilde{m}_{0}^{2}}=\frac{m^{2}}{\kappa^{2} m_{0}^{2}} .
$$

We will see that a Lorentzian effective metric arises for $\kappa^{2}>1$ (4.27), i.e. for $m^{2}<m_{0}^{2}<$ 0 . Then $H_{n}^{4}$ with $X^{a} X^{b} \eta_{a b}=-R^{2}$ (4.4) is indeed a solution of the matrix model with Lorentzian target space metric $g_{a b}(3.10)$, and $Y^{a}$ is a solution of (2.1).

One may worry about possible instabilities in the presence of negative masses. However, these mass terms are of cosmological scale, and therefore extremely small. Moreover as shown in the case of $S_{N}^{4}$ [29], even a positive bare mass term may lead to a radius stabilization at one loop, as the quantum effective action mimics a negative mass for the radial parameter(s). Thus one may hope that quantum effects stabilize the present solution even in the presence of positive but different masses. The computation of the effective metric below would then essentially go through.

Induced metric. Again we compute first the induced metric $g_{\mu \nu}$ on $H^{4} \subset \mathbb{R}^{1,4}$, which clearly has Euclidean signature at $x_{0}=R$. Using the space-like $\mathrm{SO}(4)$ symmetry, we can choose a standard reference point

$$
x=\left(x_{0}, x_{1}, 0,0,0\right)=R(\cosh (\eta), \sinh (\eta), 0,0,0) \in H^{4} \subset \mathbb{R}^{4,1}, \quad x_{0}^{2}-x_{1}^{2}=R^{2} .
$$

and use the tangential coordinate

$$
\tau=R \eta
$$

which points in the $x^{0} x^{1}$ direction,

$$
\frac{d}{d \tau} x=R(\sinh (\eta), \cosh (\eta), 0,0,0) .
$$

Then the induced metric is

$$
g_{\mu \nu}=\operatorname{diag}\left(\cosh ^{2}(\eta)-\kappa^{2} \sinh ^{2}(\eta), 1,1,1\right)
$$


in local $x^{\mu}=\left(\tau x^{2} x^{3} x^{4}\right)$ coordinates on $T_{p} \mathcal{M}$ for the standard reference point (3.16), or

$$
\begin{aligned}
d s_{g}^{2} & =R^{2}\left(\cosh ^{2}(\eta)-\kappa^{2} \sinh ^{2}(\eta)\right) d \eta^{2}+R^{2} \sinh ^{2}(\eta) d \Omega_{3} \\
& =\beta^{2}(\eta) d \eta^{2}+\alpha^{2}(\eta) d \Omega_{3}^{2}
\end{aligned}
$$

in FRW coordinates with

$$
\beta^{2}(\eta)=\frac{1}{2} R^{2}\left(\left(-\kappa^{2}+1\right) \cosh (2 \eta)+\kappa^{2}+1\right), \quad \alpha^{2}=\frac{1}{2} R^{2}(1-\cosh (2 \eta)) .
$$

Clearly $\alpha \geq 0$ vanishes only for $x^{0}=R$ where $\eta=0$. In contrast, $\beta\left(\eta_{*}\right)=0$ vanishes if

$$
\cosh \left(2 \eta_{*}\right)=\frac{\kappa^{2}+1}{\kappa^{2}-1}
$$

So again there is an interesting transition from Euclidean to Minkowski signature, but the associated singularity cannot be interpreted as Big Bang. Rather, the Big Bang will arise for the effective metric.

Effective metric. To obtain the effective metric (3.25) on $H^{4}$, we need to compute the average $\left[\theta^{a b} \theta^{c d}\right]_{S^{2}}$ for $H^{4}$. Recall that in the semi-classical limit, the $X^{a} \sim x^{a}$ provide an embedding of $H^{4}$ as a Euclidean hyperboloid in $\mathbb{R}^{1,4}$, with (anti)selfdual $\theta^{\mu \nu}$ describing an internal $S^{2}$ fiber. Therefore the averaging over this fiber is achieved as before ${ }^{12}$ via

$$
\left[\theta^{a b} \theta^{c d}\right]_{S^{2}}=\frac{1}{12} \Delta^{4}\left(P_{H}^{a c} P_{H}^{b d}-P_{H}^{b c} P_{H}^{a d} \pm \varepsilon^{a b c d e} \frac{1}{R} x^{e}\right)
$$

where now

$$
P_{H}^{a c}(x)=\eta^{a c}+\frac{1}{R^{2}} x^{a} x^{c}, \quad \eta_{a b} x^{a} x^{b}=-R^{2}
$$

is the $\mathrm{SO}(4,1)$-invariant Euclidean projector on the tangent space of $H^{4}$ (which is Euclidean w.r.t. $\left.\eta_{a b}\right)$. Note that $(4.18)$ is the unique $\mathrm{SO}(4,1)$ - invariant tensor which reflects the antisymmetry and (anti)selfduality of $\theta^{a b}$. Again we shall evaluate this at the reference point (4.11) on $H^{4}$. Then

$$
\begin{aligned}
g_{a b} P_{H}^{a b} & =g_{a b} \eta^{a b}+\frac{1}{R^{2}} g_{a b} x^{a} x^{b} \\
& =\left(\kappa^{2}+4\right)+\frac{1}{R^{2}}\left(-\kappa^{2} x_{0}^{2}+x_{0}^{2}-R^{2}\right) \\
& =\left(\kappa^{2}+3\right)-\left(\kappa^{2}-1\right) \cosh ^{2}(\eta)
\end{aligned}
$$

so that

$$
\begin{aligned}
\gamma^{b d} & =\frac{1}{12} \Delta^{4}\left(\left(g_{a b} P_{H}^{a b}\right) P_{H}^{b d}-g_{a c} P_{H}^{b c} P_{H}^{a d}\right) \\
& =: \frac{1}{12} \Delta^{4} \tilde{\gamma}_{a c} P_{H}^{b c} P_{H}^{a d}
\end{aligned}
$$

\footnotetext{
${ }^{12}$ This is the reason for using the $X^{a}$ coordinates. The Minkowskian metric $\eta^{a b}$ plays no role for this averaging.
} 
where

$$
\tilde{\gamma}_{a c}=\left(\left(\kappa^{2}+3\right)-\left(\kappa^{2}-1\right) \cosh ^{2}(\eta)\right) P_{a c}^{H}-g_{a c} .
$$

For the "undeformed" case $\kappa^{2}=1$, we recover $\tilde{\gamma}_{a c}=4 P_{a c}^{H}-g_{a c}=3 P_{a c}^{H}$. For $\eta=0$ i.e. $\left(x_{0}=R, x_{1}=0\right)$ this is Euclidean,

$$
\tilde{\gamma}_{a c}=4 P_{a c}^{H}-g_{a c}=\operatorname{diag}\left(\kappa^{2}, 3,3,3,3\right) .
$$

More generally, we compute in the local $\left(\tau x^{2} x^{3} x^{4}\right)$ coordinates

$$
\begin{aligned}
\tilde{\gamma}_{i i} & =\kappa^{2}+2-\left(\kappa^{2}-1\right) \cosh ^{2}(\eta)=\frac{1}{2}\left(\kappa^{2}+5-\left(\kappa^{2}-1\right) \cosh (2 \eta)\right) \\
& =: 3 c(\eta), \quad i=2,3,4 \\
\tilde{\gamma}_{\tau \tau} & =\left(\left(\kappa^{2}+3\right)-\left(\kappa^{2}-1\right) \cosh ^{2}(\eta)\right) P_{\tau \tau}^{H}-g_{\tau \tau} \\
& =3
\end{aligned}
$$

since $P_{\tau \tau}^{H}=1$ and

$$
\begin{aligned}
g_{\tau \tau} & =(\sinh (\eta), \cosh (\eta)) \operatorname{diag}\left(-\kappa^{2}, 1\right)(\sinh (\eta), \cosh (\eta)) \\
& =\kappa^{2}+\left(-\kappa^{2}+1\right) \cosh ^{2} \eta
\end{aligned}
$$

Therefore $\partial_{\tau}$ is always space-like, and

$$
\gamma^{\mu \nu}=\frac{1}{4} \Delta^{4}(1, c(\eta), c(\eta), c(\eta))
$$

in the above coordinates. This is consistent with $x^{i}=0$ since $c(0)=1$. The effective metric changes signature at $c\left(\eta_{0}\right)=0$ i.e.

$$
\cosh \left(2 \eta_{0}\right)=\frac{\kappa^{2}+5}{\kappa^{2}-1}
$$

provided $\kappa^{2}>1$, and the metric is Lorentzian for $\eta>\eta_{0}$ with $c(\eta)<0$. Again, this happens after the signature change for the induced metric (4.17), as indicated in figure 3. From a target space point of view, the size of the universe at the BB may or may not be large depending on $\kappa$, but it is small in the effective metric.

In the same coordinates, the $\mathrm{SO}(1,4)$-invariant volume form $\sqrt{\left|\theta^{\mu \nu}\right|} \sim \frac{\Delta^{4}}{4}$ is constant. Hence the conformal factor (3.25) is

$$
\alpha=\sqrt{\frac{\left|\theta^{\mu \nu}\right|}{\left|\gamma^{\mu \nu}\right|}}=\frac{4}{\Delta^{4}}|c(\eta)|^{-3 / 2}
$$

and the effective metric is obtained as

$$
G_{\mu \nu}=|c(\eta)|^{3 / 2} \operatorname{diag}\left(1, \mathrm{c}(\eta)^{-1}, \mathrm{c}(\eta)^{-1}, \mathrm{c}(\eta)^{-1}\right) .
$$




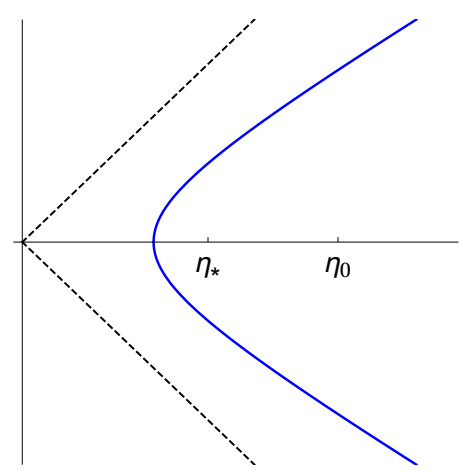

Figure 3. Schematic picture of the expanding universe with lightcone for $g_{\mu \nu}$, indicating $\eta_{*}$ and $\eta_{0}$.

Scale factor. Extracting the cosmological evolution proceeds as in section 3.2. We express the metric in FRW coordinates,

$$
d s_{G}^{2}=b^{2}(\eta) d \eta^{2}-\tilde{a}^{2}(\eta) d \Omega^{2}=d t^{2}-a^{2}(t) d \Omega^{2}
$$

where $d \Omega^{2}$ is the length element on a spatial 3 -sphere $S^{3}$ with unit radius. Thus

$$
\tilde{a}^{2}(\eta)=R^{2}|c(\eta)|^{1 / 2}=a^{2}(t), \quad b^{2}(\eta)=R^{2}|c(\eta)|^{3 / 2}
$$

in the cosmological era (with Minkowski signature). Again both $a$ and $b$ vanish at the time $\eta_{0}$ of the $\mathrm{BB}$, in contrast to the induced metric. We set $R=1$ for simplicity. Then

$$
b=a^{3} .
$$

The comoving time parameter $t$ is determined from

$$
\dot{\eta}=b^{-1}=a^{-3} .
$$

We can solve this again in the cosmological era

$$
a^{4}=|c(\eta)|=\frac{1}{6}\left(-\left(\kappa^{2}+5\right)+\left(\kappa^{2}-1\right) \cosh (2 \eta)\right),
$$

which gives

$$
\dot{a}=\frac{1}{12} a^{-6} \sqrt{\left(\kappa^{2}+5+6 a^{4}\right)^{2}-\left(\kappa^{2}-1\right)^{2}} .
$$

This shows the same initial $a \sim t^{1 / 7}$ expansion as in (3.47). However for large $t$, we obtain

$$
\dot{a} \approx \frac{1}{2} a^{-2}
$$

so that

$$
a(t) \sim t^{1 / 3}
$$



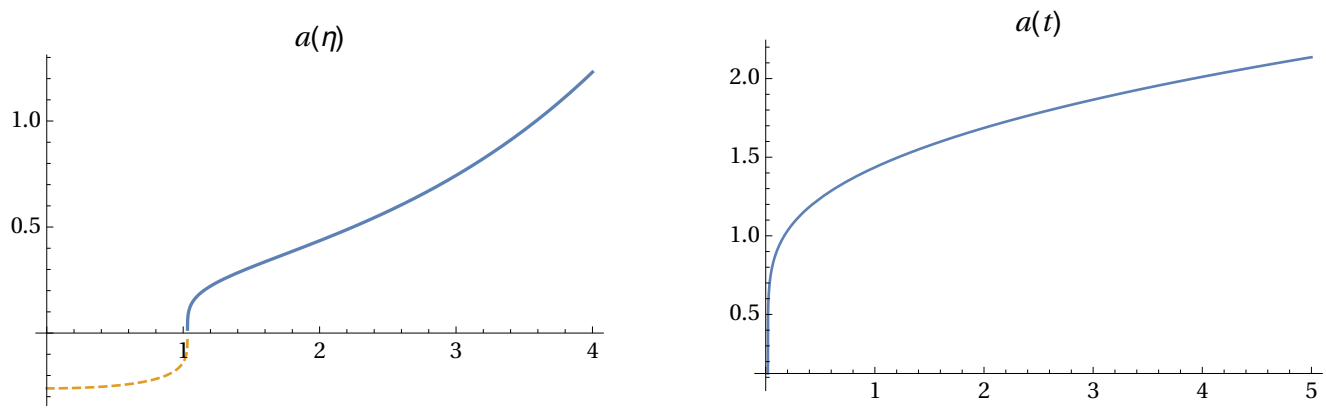

Figure 4. $a(\eta)$ and $a(t)$ for the $H^{4}$ cosmology with $\kappa^{2}=3$. The red dashed line is the Euclidean era with imaginary $a(\eta)$.

for the late-time evolution. The expansion is somewhat slower than for a matter-dominated universe, which would be $a(t) \sim t^{2 / 3}$. The Hubble parameter for large $t$ is

$$
H=\frac{\dot{a}}{a} \approx \frac{1}{2} a^{-3} \sim t^{-1}
$$

and it is again decelerating at all times, $\ddot{a}<0$.

To illustrate the expansion, we plot $a(\eta)$ as well as $a(t)$ in figure 4 . From the target space point of view, the $\mathrm{BB}$ singularity of $a(\eta)$ is milder than in the $t$ variables, but still manifest. Again, the scale parameter $a(\eta)$ is imaginary before the BB for $0 \leq \eta<\eta_{0}$, covering the entire $H^{4}$. Accordingly, the effective metric is Euclidean for $\eta<\eta_{0}$. The apparent acceleration of $a(\eta)$ in figure 4 is however an artifact, and there is no acceleration in the comoving time $a(t)$. Nevertheless, it suggests that some mild corrections of the metric may easily modify this conclusion; see e.g. [13] for a related discussion in 2 dimensions. Some other aspects of this solutions will be discussed below.

\subsection{Outlook}

Excitation modes. The above solutions define not only geometrical space-times $\mathcal{M}$; the bosonic and fermionic excitation modes on these backgrounds in the matrix model define gauge theories living on $\mathcal{M}$. These fluctuations can be understood in terms of the noncommutative algebra of functions, which is $\operatorname{End}(\mathcal{H}) \sim F u n\left(\mathbb{C} P^{1,2}\right)$ for $H_{n}^{4}$, and $\operatorname{End}(\mathcal{H}) \sim \operatorname{Fun}\left(\mathbb{C} P^{3}\right)$ for $S_{N}^{4}$. Since these are equivariant ("twisted") bundles over $\mathcal{M}^{4}$, the harmonics on the fiber $S^{2}$ lead to higher spin modes on $\mathcal{M}$ (in contrast to Kaluza-Klein modes which arise on ordinary compactifications). Explicitly, functions $\Phi \in \operatorname{End}\left(\mathcal{H}_{n}\right)$ can be expanded in the form

$$
\Phi=\phi(x)+\phi_{a b}(x) \mathcal{M}^{a b}+\ldots
$$

This amounts to a decomposition into higher spin modes as in [10, 45], whose propagation is governed by $\square_{Y}(2.3)$, hence by the effective metric $G_{\mu \nu}$. In particular, the tangential fluctuations $Y^{\mu}+\mathcal{A}^{\mu}$ include the modes

$$
\mathcal{A}^{\mu}=\theta^{\mu \nu} h_{\nu \rho}(x) P^{\rho}
$$


where $P^{\mu} \in \mathfrak{s o}(4,1)$ is the local generator of translations, cf. [10]. The symmetric part of $h_{\mu \nu}$ could naturally play the role of the spin 2 graviton. Whether or not this leads to an acceptable gravity will be examined elsewhere.

De Sitter and other solutions. It is natural to wonder about de Sitter branes. There are indeed candidates for fuzzy de Sitter space based on the principal series representations of $\mathrm{SO}(4,1)$ [12, 32, 39-42], some of which should be solutions of the matrix model for $m_{0}^{2}=m^{2}$; see also [43] for related work. However then the effective metric would coincide with the induced one, without BB. Even for $m_{0}^{2} \neq m^{2}$, it is hard to see how a BB might arise in this case. Therefore we will not consider this case in the present paper. There are also mathematical issues, such as the expected non-compact nature of the internal space, which makes the averaging procedure problematic for de Sitter-type spaces.

It may also be possible to find a twisted embedding along the lines of [44] to obtain a matrix realization of a covariant space-time with big bounce. ${ }^{13}$

\section{Discussion}

We presented a novel and simple mechanism how a cosmological Big Bang could arise in the context of Yang-Mills matrix models. The BB arises from a signature change in the effective metric on noncommutative space-time branes embedded in Lorentzian target space, taking into account the quantized 4 -volume form. The underlying brane is completely regular, at least in the present simplified treatment. The rapid initial expansion arises from a singular conformal factor, which follows from the quantized flux in conjunction with the signature change. There is a period "before" the BB where the effective metric is Euclidean but the embedding ("closed string") metric still has Minkowski signature. One may hope that this helps to avoid the horizon problem, possibly even in the absence of exponential inflation. The initial sector of the brane is a Euclidean cap. This is somewhat reminiscent of an instanton, ${ }^{14}$ however the path integral (2.4) is always over $e^{i S}$.

Note that the mechanism does not apply to more traditional brane-world scenarios, where the effective metric is the induced (pull-back) metric from the target space metric. In such a scenario, a signature change would not lead to a rapid initial expansion.

The late-time behavior found in the solutions under consideration is different from the currently accepted $\Lambda \mathrm{CDM}$ model, even for the solution based on $H^{4}$ which is expanding forever. For example, we can compute the age of universe in terms of the present Hubble parameter, assuming that the early phase of the universe is negligible:

$$
t=\int \frac{d a}{a H(a)}=\frac{1}{3 H(t)} .
$$

This deviates from the accepted values by a factor $\approx 3$. Also, equation (4.38) is rather strange compared with the Friedmann equations. On the other hand, the analysis of the

\footnotetext{
${ }^{13}$ Note that the mechanism in [44] is different from the present one and essentially relies on the embedding metric, which can be the effective one only assuming a certain complexification.

${ }^{14}$ This aspect is somewhat reminiscent of Vilenkin's "tunneling from nothing" proposal [20]. I would like to thank H. Kawai for pointing this out.
} 
model is very crude: the influence of matter or radiation, and even gravity in the ordinary sense, are completely ignored. It is therefore remarkable that semi-realistic cosmologies including a BB nevertheless arise, without any reference to the Friedmann equations and GR. Hence basic cosmology might have a simple and robust origin in this scenario.

Of course a space-time by itself does not provide a full cosmology. To obtain interesting physics, gravity must of course be present, at least for intermediate scales. As explained in section 4.3, spin 2 modes which could play the role of gravitons do indeed arise, however this needs to be re-examined carefully for the present Lorentzian backgrounds. ${ }^{15}$ Along with the other excitation modes, this will clearly affect the expansion of the universe. Loop corrections will also modify the geometry of the brane solution, e.g. via corrections to the mass parameters in the model, which might even depend on time. Fuzzy extra dimensions realized along the lines of [28] may also affect the expansion. Finally, the BB entails high temperatures, which will certainly have an impact on the early expansion. Therefore the quantitative results should be taken with much caution.

There is also a more basic issue which needs to be clarified. We have determined the conformal factor of the metric by matching the kinetic term with the standard covariant metric form (3.23). If we would repeat this procedure for a naive mass term, we would obtain a different conformal factor. This may be reconciled noting that the mass terms for matter should in fact not arise from the bare matrix model but from spontaneous symmetry breaking as in the standard model, which would presumably lead to a consistent picture. Therefore the present approach seems justified; note also that there is no issue for gauge fields due to conformal invariance. Nevertheless, the treatment of the conformal factor may need some refinement, which could have a non-trivial effect on the late-time cosmology.

From a more formal perspective, the present solutions are also very interesting. In particular, the solutions provide simple examples for a homogeneous and isotropic quantum space-times with Minkowski signature, with intrinsic IR and UV cutoff ${ }^{16}$ in the $S_{N}^{4}$ case, and a UV cutoff in the $H_{n}^{4}$ case. Hence the mathematical tools and techniques for field theory on such a space can be worked out, including the appropriate boundary conditions and the $i \varepsilon$ prescription for loop integrals. This would allow to compute quantum corrections to the geometry as well as for field theory in a clear-cut way, notably for the supersymmetric IKKT model. In particular, the stabilization mechanism in [29] should apply in some way. On the other hand, the $H_{n}^{4}$ solution is perhaps the most reasonable noncommutative cosmological solution available up to now, and it is very promising from the point of view of emergent gravity, due to the presence of spin 2 modes.

In summary, the main message of this paper is a conceptually very appealing mechanism for a Big Bang within the matrix model, based on a quantum structure of space-time. A large universe arises quite naturally, determined only by a discrete choice of the representation, as well as a parameter $\kappa$ which can be of order 1 . The mechanism is robust and essentially classical (unlike e.g. Vilenkin's "universe from nothing" proposal [20] for the

\footnotetext{
${ }^{15}$ While for undeformed $S_{N}^{4}$ this does not appear to be realistic [45], the case of deformed $H_{n}^{4}$ with small $n$ looks very promising.

${ }^{16}$ There is some superficial similarity with [46], however that approach is still based on the infinitedimensional algebra of functions on a classical Euclidean manifold.
} 
BB), and does not rely on general relativity. However, a more detailed understanding of the associated physics is required, which needs much more work.

\section{Acknowledgments}

I would like to thank in particular Hikaru Kawai and Kentaroh Yoshida for very useful discussions, hospitality and support during a stay at Kyoto University, where part of this work was done. I would also like to thank J. Nishimura and M. Sperling for useful discussions. This work was made possible by the Austrian Science Fund (FWF) grant P28590. The Action MP1405 QSPACE from the European Cooperation in Science and Technology (COST) also provided support in the context of this work.

\section{A No finite-dimensional solutions of massless Lorentzian Yang-Mills ma- trix models}

We show the following simple result, which explains the presence of $m^{2}$ in (2.2):

Lemma. Let $\square_{X}=\eta_{a b}\left[X^{a},\left[X^{b},.\right]\right]$ for $\eta_{a b}=\operatorname{diag}(-1,1, \ldots, 1)$. Assume that $X^{0}, X^{i}$ are finite-dimensional hermitian matrices which are solution of

$$
\square_{X} X^{0}=0=\square_{X} X^{i} .
$$

Then all matrices $X_{i}, X_{0}$ commute with each other.

Proof. The eom for $X^{0}$ implies

$$
-\operatorname{tr}\left(X^{0} \square_{X} X^{0}\right)=0=\sum_{i} \operatorname{tr}\left(\left(\left[X^{0}, X^{i}\right]\right)^{2}=\sum_{i} \operatorname{tr} A_{i}^{2}\right.
$$

where $A_{i}:=i\left[X_{0}, X_{i}\right]=A_{i}^{\dagger}$ is hermitian. It follows that

$$
0=\operatorname{tr} A_{i}^{2} \quad \forall i
$$

and therefore $A_{i}=0$. This means that

$$
\left[X_{0}, X_{i}\right]=0
$$

Then the equations of motion for $X_{i}$ imply

$$
\pm \operatorname{tr} X^{i} \square_{X} X^{i}=0=\sum_{j} \operatorname{tr}\left(\left(\left[X^{j}, X^{i}\right]\right)^{2} \quad \text { for each } i .\right.
$$

This implies

$$
\left[X_{i}, X_{j}\right]=0 \quad \forall i, j
$$

as before, and all matrices commute.

However, there are finite-dimensional non-commutative solutions in the presence of masses, as illustrated in this paper. 
Open Access. This article is distributed under the terms of the Creative Commons Attribution License (CC-BY 4.0), which permits any use, distribution and reproduction in any medium, provided the original author(s) and source are credited.

\section{References}

[1] N. Ishibashi, H. Kawai, Y. Kitazawa and A. Tsuchiya, A large-N reduced model as superstring, Nucl. Phys. B 498 (1997) 467 [hep-th/9612115] [INSPIRE].

[2] I. Chepelev and A.A. Tseytlin, Interactions of type IIB D-branes from D instanton matrix model, Nucl. Phys. B 511 (1998) 629 [hep-th/9705120] [INSPIRE].

[3] M.R. Douglas and W. Taylor, Branes in the bulk of Anti-de Sitter space, hep-th/9807225 [INSPIRE].

[4] H.C. Steinacker, String states, loops and effective actions in noncommutative field theory and matrix models, Nucl. Phys. B 910 (2016) 346 [arXiv:1606.00646] [INSPIRE].

[5] H. Aoki et al., Noncommutative Yang-Mills in IIB matrix model, Nucl. Phys. B 565 (2000) 176 [hep-th/9908141] [INSPIRE].

[6] R.J. Szabo, Quantum field theory on noncommutative spaces, Phys. Rept. 378 (2003) 207 [hep-th/0109162] [INSPIRE].

[7] S. Minwalla, M. Van Raamsdonk and N. Seiberg, Noncommutative perturbative dynamics, JHEP 02 (2000) 020 [hep-th/9912072] [INSPIRE].

[8] Y. Kinar, G. Lifschytz and J. Sonnenschein, UV/IR connection: A Matrix perspective, JHEP 08 (2001) 001 [hep-th/0105089] [INSPIRE].

[9] H. Steinacker, Emergent geometry and gravity from matrix models: an introduction, Class. Quant. Grav. 27 (2010) 133001 [arXiv:1003.4134] [INSPIRE].

[10] H.C. Steinacker, Emergent gravity on covariant quantum spaces in the IKKT model, JHEP 12 (2016) 156 [arXiv:1606.00769] [INSPIRE].

[11] N. Seiberg and E. Witten, String theory and noncommutative geometry, JHEP 09 (1999) 032 [hep-th/9908142] [INSPIRE].

[12] D. Jurman and H. Steinacker, $2 D$ fuzzy Anti-de Sitter space from matrix models, JHEP 01 (2014) 100 [arXiv:1309.1598] [INSPIRE].

[13] A. Chaney, L. Lu and A. Stern, Matrix model approach to cosmology, Phys. Rev. D 93 (2016) 064074 [arXiv:1511.06816] [INSPIRE].

[14] H. Steinacker, Split noncommutativity and compactified brane solutions in matrix models, Prog. Theor. Phys. 126 (2011) 613 [arXiv:1106.6153] [InSPIRE].

[15] S.-W. Kim, J. Nishimura and A. Tsuchiya, Late time behaviors of the expanding universe in the IIB matrix model, JHEP 10 (2012) 147 [arXiv:1208.0711] [INSPIRE].

[16] S.-W. Kim, J. Nishimura and A. Tsuchiya, Expanding $(3+1)$-dimensional universe from a Lorentzian matrix model for superstring theory in $(9+1)$-dimensions, Phys. Rev. Lett. 108 (2012) 011601 [arXiv:1108.1540] [INSPIRE].

[17] S.-W. Kim, J. Nishimura and A. Tsuchiya, Expanding universe as a classical solution in the Lorentzian matrix model for nonperturbative superstring theory, Phys. Rev. D 86 (2012) 027901 [arXiv: 1110.4803 ] [INSPIRE]. 
[18] A. Chaney, L. Lu and A. Stern, Lorentzian fuzzy spheres, Phys. Rev. D 92 (2015) 064021 [arXiv: 1506.03505] [INSPIRE].

[19] A. Chaney and A. Stern, Fuzzy CP $P^{2}$ spacetimes, Phys. Rev. D 95 (2017) 046001 [arXiv: 1612.01964] [INSPIRE].

[20] A. Vilenkin, Creation of universes from nothing, Phys. Lett. B 117 (1982) 25.

[21] M. Hanada and H. Shimada, On the continuity of the commutative limit of the $4 d N=4$ non-commutative super Yang-Mills theory, Nucl. Phys. B 892 (2015) 449 [arXiv:1410.4503] [INSPIRE].

[22] H. Grosse, C. Klimčík and P. Prešnajder, On finite $4 D$ quantum field theory in noncommutative geometry, Commun. Math. Phys. 180 (1996) 429 [hep-th/9602115] [INSPIRE].

[23] J. Castelino, S. Lee and W. Taylor, Longitudinal five-branes as four spheres in matrix theory, Nucl. Phys. B 526 (1998) 334 [hep-th/9712105] [INSPIRE].

[24] S. Ramgoolam, On spherical harmonics for fuzzy spheres in diverse dimensions, Nucl. Phys. B 610 (2001) 461 [hep-th/0105006] [INSPIRE].

[25] P.-M. Ho and S. Ramgoolam, Higher dimensional geometries from matrix brane constructions, Nucl. Phys. B 627 (2002) 266 [hep-th/0111278] [INSPIRE].

[26] Y. Kimura, Noncommutative gauge theory on fuzzy four sphere and matrix model, Nucl. Phys. B 637 (2002) 177 [hep-th/0204256] [INSPIRE].

[27] J. Medina and D. O'Connor, Scalar field theory on fuzzy S', JHEP 11 (2003) 051 [hep-th/0212170] [INSPIRE].

[28] M. Sperling and H.C. Steinacker, Covariant 4-dimensional fuzzy spheres, matrix models and higher spin, J. Phys. A 50 (2017) 375202 [arXiv:1704.02863] [INSPIRE].

[29] H.C. Steinacker, One-loop stabilization of the fuzzy four-sphere via softly broken SUSY, JHEP 12 (2015) 115 [arXiv: 1510.05779] [INSPIRE].

[30] H. Steinacker, Non-commutative geometry and matrix models, PoS (QGQGS 2011) 004 [arXiv:1109.5521] [INSPIRE].

[31] T. Azuma, S. Bal, K. Nagao and J. Nishimura, Absence of a fuzzy $S^{4}$ phase in the dimensionally reduced 5D Yang-Mills-Chern-Simons model, JHEP 07 (2004) 066 [hep-th/0405096] [INSPIRE].

[32] K. Hasebe, Non-compact Hopf maps and fuzzy ultra-hyperboloids, Nucl. Phys. B 865 (2012) 148 [arXiv: 1207.1968] [INSPIRE].

[33] H. Grosse, P. Prešnajder and Z. Wang, Quantum field theory on quantized Bergman domain, J. Math. Phys. 53 (2012) 013508 [arXiv: 1005.5723] [InSPIRE].

[34] S. Fernando and M. Günaydin, Minimal unitary representation of $\mathrm{SU}(2,2)$ and its deformations as massless conformal fields and their supersymmetric extensions, J. Math. Phys. 51 (2010) 082301 [arXiv:0908.3624] [INSPIRE].

[35] M. Günaydin, D. Minic and M. Zagermann, $4 D$ doubleton conformal theories, CPT and IIB string on $A d S_{5} \times S^{5}$, Nucl. Phys. B 534 (1998) 96 [Erratum ibid. B 538 (1999) 531] [hep-th/9806042] [INSPIRE].

[36] G. Mack, All unitary ray representations of the conformal group $\mathrm{SU}(2,2)$ with positive energy, Commun. Math. Phys. 55 (1977) 1 [INSPIRE]. 
[37] G. Mack and I. Todorov, Irreducibility of the ladder representations of $u(2,2)$ when restricted to the Poincaré subgroup, J. Math. Phys. 10 (1969) 2078 [InSPIRE].

[38] W. Heidenreich, Tensor products of positive energy representations of $\mathrm{SO}(3,2)$ and $\mathrm{SO}(4,2)$, J. Math. Phys. 22 (1981) 1566 [inSPIRE].

[39] M. Burić, D. Latas and L. Nenadovic, Fuzzy de Sitter space, arXiv:1709.05158 [InSPIRE].

[40] M. Burić and J. Madore, Noncommutative de Sitter and FRW spaces, Eur. Phys. J. C 75 (2015) 502 [arXiv: 1508.06058] [INSPIRE].

[41] J.-P. Gazeau, J. Mourad and J. Queva, Fuzzy de Sitter space-times via coherent states quantization, quant-ph/0610222 [INSPIRE].

[42] J.-P. Gazeau and F. Toppan, A natural fuzzyness of de Sitter space-time, Class. Quant. Grav. 27 (2010) 025004 [arXiv:0907.0021] [INSPIRE].

[43] J. Heckman and H. Verlinde, Covariant non-commutative space-time, Nucl. Phys. B 894 (2015) 58 [arXiv: 1401.1810] [INSPIRE].

[44] D. Klammer and H. Steinacker, Cosmological solutions of emergent noncommutative gravity, Phys. Rev. Lett. 102 (2009) 221301 [arXiv:0903.0986] [INSPIRE].

[45] M. Sperling and H.C. Steinacker, Higher spin gauge theory on fuzzy $S_{N}^{4}$, J. Phys. A 51 (2018) 075201 [arXiv: 1707.00885] [INSPIRE].

[46] A.H. Chamseddine, A. Connes and V. Mukhanov, Quanta of geometry: noncommutative aspects, Phys. Rev. Lett. 114 (2015) 091302 [arXiv:1409.2471] [INSPIRE]. 